\title{
Metodologia para a deteção de artefactos luminosos em imagens de retinografia com aplicação em rastreio oftalmológico
}

\author{
André Damas Mora ${ }^{1,2}$, José Manuel Fonseca ${ }^{1,2}$ \\ atm@uninova.pt,jmf@uninova.pt \\ ${ }^{1}$ Centro de Tecnologias e Sistemas - Uninova, Campus da FCT-UNL, 2829-517 Caparica, Portugal \\ 2 Departamento de Eng. Electrotécnica, FCT-UNL, Campus da FCT-UNL, 2829-516 Caparica, Portugal
}

DOI: 10.4304/risti.13.51-63

\begin{abstract}
Resumo: O diagnóstico automático de doenças da retina baseado em processamento de imagem vê frequentemente a sua precisão comprometida pela dificuldade intrínseca na deteção de estruturas anormais e por deficiências na aquisição de imagem. Em cenários de rastreio, estas deficiências podem levar a que um número significativo de imagens tenham de ser repetidas, implicando custos e ineficiência do sistema. Neste artigo propõe-se uma metodologia que avalia de forma automática a qualidade das imagens captadas possibilitando ao operador repetir a aquisição caso se justifique. O método proposto identifica diferentes tipos de artefactos com base na sua forma, cor e intensidade. Utilizando um conjunto de 61 imagens foi obtida uma sensibilidade de $97 \%$ com 0.12 falsos positivos por imagem na deteção do artefacto central e $73 \%$ de sensibilidade com 0.36 de falsos positivos para os reflexos luminosos. Estes resultados podem considerar-se positivos tendo em conta a baixa qualidade e a heterogeneidade das imagens processadas.
\end{abstract}

Palavras-chave: classificação automática; processamento de imagem; imagem da retina; reflexos luminosos; rastreio oftalmológico.

\section{Methodology for image artifacts detection in retinal images with application in Ophthalmology Screening}

Abstract: Automatic diagnostic systems for retinal diseases based on image processing have continuously demonstrated its potential for clinical practice. However, their accuracy is often compromised by the inherent difficulty in detecting abnormal structures and aggravated by deficiencies in image acquisition. In screening scenarios, these deficiencies can lead to a significant amount of repeated images, implying costs and system's inefficiency. In this paper we propose a methodology to automatically evaluate the quality of captured images allowing the operator to repeat the acquisition if appropriate. The proposed method identifies different types of artefacts based on color, shape and image intensity. Using a set of 61 images a sensitivity of $97 \%$ at a rate of 0.12 false 
positives in the central artifact detection and $73 \%$ sensitivity with 0.36 false positives on the detection of light flares, were obtained. These results can be considered positive given the poor quality and heterogeneity of the processed images.

Keywords: Automatic classification; image processing; retinal images; light flares; ophthalmology screening.

\section{Introdução}

O progressivo envelhecimento da população mundial (United Nations, 2013) associado às diversas patologias oculares relacionadas com a idade (Porte, 2012) leva a que a importância destas doenças tenha vindo a aumentar constantemente nas últimas décadas. Uma vez que a visão é fundamental para uma boa qualidade de vida, a deteção precoce de doenças oculares é de grande importância especialmente porque muitas patologias são assintomáticas até atingirem um estado avançado de desenvolvimento em que são muitas vezes irreversíveis (Rogers, 2007). Para efectuar a deteção precoce na generalidade da população é necessário realizar acções de rastreio em que um grande número de indivíduos é observado sendo efetuada a captação de imagens de fundoscopia que são posteriormente avaliadas por especialistas.

No entanto, as imagens da retina captadas por câmaras de fundoscopia podem conter artefactos que podem implicar a sua inutilização ou conduzir a diagnósticos incorretos especialmente quando as patologias são detetadas por métodos automáticos (Azemin et al., 2011; M. Niemeijer et al., 2007; Wilson et al., 2003). Os artefactos mais comuns são provocados por dedadas, pó e manchas causadas por tosse ou espirros do paciente (Hamilton et al., 1998). Estes artefactos são reconhecíveis como pontos de maior intensidade espalhados pela imagem (figura 1). A sujidade no interior do sistema ótico (que pode ser originada por ações de manutenção tais como mudança de lâmpadas) pode também causar imperfeições significativas na imagem (Hamilton et al., 1998) criando um anel luminoso no centro da imagem da retina a que se dá o nome de artefacto central. A aquisição de imagens da retina é efetuada com base em diversos processos de refração, reflexão e absorção da luz que transita entre o olho e o detector. A obstrução ou desalinhamento deste processo complexo leva inevitavelmente à produção de uma imagem defeituosa.

Quando existe desalinhamento, a imagem apresenta grandes zonas de alta intensidade luminosa com o surgimento de diversas cores (figura 1.b). A distância entre o olho e o sistema de aquisição é também de grande importância. Se a distância for superior ao desejável a imagem resultante será desfocada, enquanto que se a distância for demasiado pequena a imagem poderá apresentar reflexos indesejados que perturbarão a sua qualidade (Hamilton et al., 1998). 

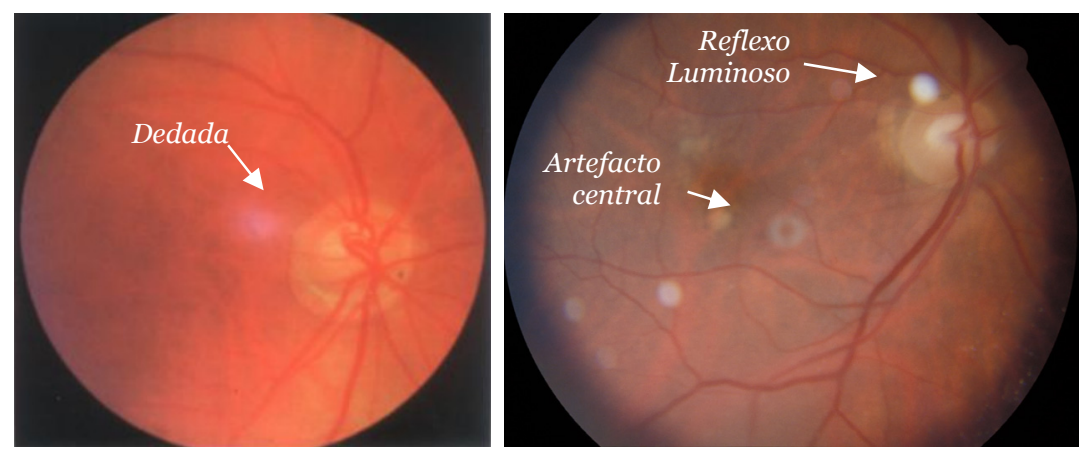

Figura 1 - Exemplos de artefactos em imagens de fundoscopia.

a) dedada (Hamilton et al., 1998); b) reflexos luminosos e artefacto central

Os artefactos mais comuns são os reflexos luminosos (light flares) que podem ser observados na figura 1.b. Como estes artefactos são causados pela reflexão da luz ou pela sujidade ao longo do sistema ótico, podem variar em número e intensidade (Burke, 1996). Apesar de poder parecer inofensiva, a ocorrência de artefactos nas imagens de fundoscopia pode ter um impacto significativo nos resultados dos sistemas de diagnóstico automático (Davis et al., 2009; Mora et al., 2011; Pinão et al., 2013; Yu et al., 2012) podendo as áreas da imagem com alta ou baixa luminosidade ser facilmente confundidas com patologias, aumentando assim o número de falsos positivos.

A deteção automática de artefactos luminosos em imagens da retina é um tema pouco explorado pela comunidade científica. No entanto, o seu estudo é importante pois permitirá melhorar a eficácia das campanhas de rastreio ao permitir a deteção de deficiências. Se efectuada no momento da aquisição da imagem, a deteção de artefactos pode levar à repetição imediata do exame evitando posteriores recolhas. Caso a detecção seja aplicada após a campanha de rastreio, terá como principal utilidade evitar a utilização da imagem nas zonas assinaladas como artefactos. A ocorrência de artefactos luminosos ocorre também noutros cenários de aplicação onde já foram testadas algumas técnicas para a sua deteção. Alguns trabalhos (Gu et al., 2009; Zhou \& Lin, 2007) propuseram algoritmos que se baseiam na aquisição de múltiplas imagens de modo a conhecer o modelo das distorções provocadas para em seguida corrigir as alterações enquanto outros propõem algoritmos para corrigir as reflexões através da informação circundante à reflexão (Bertalmio et al., 2000; Sun et al., 2005). Contudo estes métodos não são aplicáveis nas imagens de retina uma vez que apenas se dispõe de imagens isoladas e porque na grande maioria dos casos os reflexos provocam alterações irreversíveis na imagem não sendo possível recuperar a informação mascarada pelo artefacto.

Neste artigo apresentamos um novo método para a deteção de artefactos em imagens da retina que pode ser utilizado para controlo automático de qualidade na captação de imagens especialmente em situações de rastreio ou na seleção das imagens para avaliação automática de patologias de forma a evitar a análise de imagens com artefactos que podem produzir falsos positivos. 
O artigo começará por apresentar as várias fases da metodologia, nomeadamente de preprocessamento de imagem e de detecção de artefactos, para em seguida apresentar resultados da aplicação da metodologia a um conjunto de imagens recolhidas para um estudo ciêntifico que visou relacionar o tratamento de cataratas com a degeneração macular relacionada com a idade (DMRI).

\section{Métodos}

Entre os diversos artefactos luminosos que podem surgir em imagens de fundos, os reflexos luminosos e o artefacto central são os mais comuns (Hamilton et al., 1998). Os reflexos luminosos são normalmente manchas arredondadas que podem variar em cor e tamanho. $\mathrm{O}$ chamado artefacto central é consequência da acumulação de sujidade no sistema ótico apresentando forma toroidal, sempre centrado na imagem (donut shape).

Apesar da diferente natureza destes dois tipos de artefactos, a metodologia de base proposta para a sua deteção é baseada no mesmo algoritmo de comparação de padrões (template matching), utilizando diferentes configurações para cada um deles. Dado que o método de deteção automática tem tendência para produzir uma sobresegmentação da imagem foi criado um processo de pós-classificação baseado em árvores de decisão para eliminar as zonas indevidamente segmentadas.

As secções seguintes apresentam detalhadamente a metodologia proposta desde o préprocessamento da imagem até à classificação final dos artefactos detetados.

\subsection{Imagens utilizadas}

As 61 imagens da retina centradas na zona macular utilizadas neste estudo foram obtidas a partir do trabalho efetuado por Simmon Brunner "ECAM - Monitoring Drusen and Geographic Atrophy after Cataract Surgery Study" (Brunner et al., 2013). Todas as imagens foram captadas no Departmento de Oftalmologia do Rudolfstiftung Hospital, Vienna, Austria usando um retinógrafo de $40^{\circ}$ (Canon CR2-45NM, Veatch Ophthalmic Instruments) e digitalizadas com uma resolução de 1639x1116 pixéis. Os pacientes que participaram neste estudo sofriam de cataratas e de degeneração macular relacionada com a idade (DMRI), apresentando uma idade média de 80.5 \pm 6 anos.

De forma a permitir avaliar a precisão da deteção automática de artefactos um perito analisou manualmente as 61 imagens tendo detectado 96 reflexos luminosos e 50 artefactos centrais. É de notar que, uma vez que os pacientes que participaram neste estudo sofriam de cataratas (o que obviamente aumenta a opacidade e a distorção de cor das imagens captadas), algumas das imagens apresentam uma qualidade bastante reduzida.

\subsection{Pré-processamento}

O primeiro passo da metodologia proposta é o melhoramento do contraste entre os artefactos e o fundo da imagem. Foram analisados os espaços de cor RGB e HSV, verificando-se que o espaço HSV permite um melhor contraste para a detecção de artefactos luminosos. Analisando os três canais RGB podemos constatar que o canal vermelho apresenta um contraste reduzido entre os artefactos e o fundo, enquanto que 
os canais verde e azul são semelhantes entre si apresentando ambos melhor contraste que o vermelho (com ligeira vantagem para o canal azul - figura. 2.a). Entre os três canais HSV, apenas o canal de saturação é útil para a detecção de artefactos. Embora neste caso a imagem pareça demasiado clara podemos verificar a existência de um bom contraste entre os artefactos e o fundo da imagem (figura 2.b). O canal de saturação do espaço de cor HSV foi portanto o escolhido para a detecção dos artefactos.
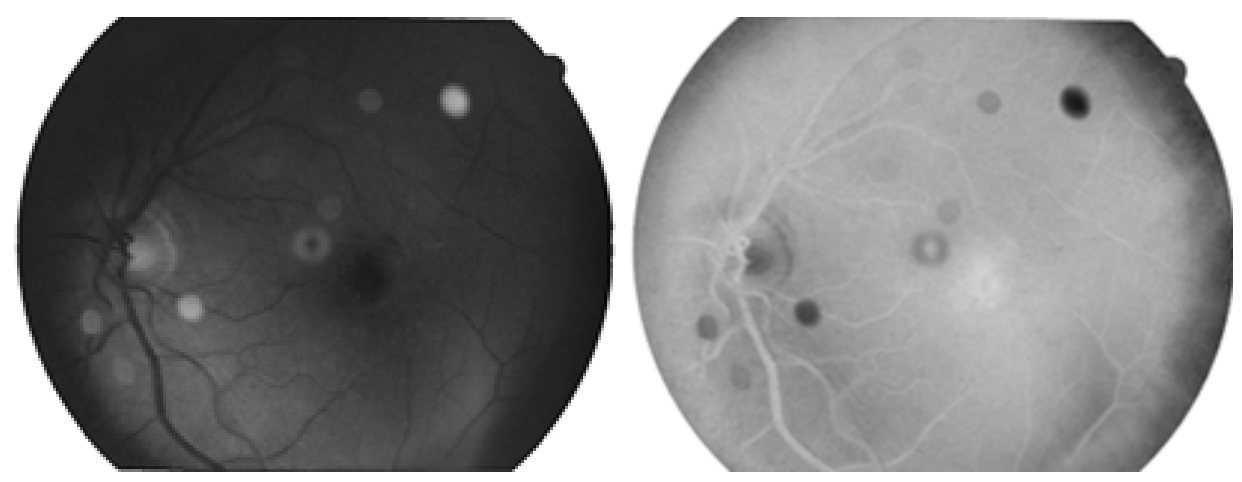

Figura 2 - Comparação de canais de cor de uma imagem de retina;

a) canal azul; b) canal de saturação.

\subsection{Deteção de Reflexos Luminosos}

Para a deteção dos reflexos luminosos é aplicada a técnica de comparação de padrões sendo utilizado o canal da saturação. Neste caso, a deteção de reflexos será baseada na sua forma e tamanho.

A técnica de comparação de padrões requer a existência de uma imagem padrão que se assemelhe à estrutura a procurar, sendo esta normalmente de dimensão inferior à imagem onde será efectuada a pesquisa. A pesquisa é efetuada deslocando a imagem padrão $(T(x, y))$ sobre a imagem original $(I(x, y))$ e em cada uma das localizações $\left(x^{\prime}, y^{\prime}\right)$ comparar pixel a pixel as duas imagens, com vista à determinação de um valor de similaridade. Neste trabalho foi utilizado como medida de similaridade o Coeficiente de Correlação Normalizado $\left(R_{c c \_n o r m}\left(x^{\prime}, y^{\prime}\right)\right)(1)$ que pelo facto de remover o valor médio da intensidade (2) da imagem original e da imagem padrão e por normalizar o seu valor, apresenta uma boa tolerância a variações de iluminação e de contraste. O resultado da aplicação do método é uma imagem como a apresentada na figura 3.c, onde as regiões que mais se assemelham ao padrão têm uma maior intensidade.

$$
R_{c c_{-} n o r m}\left(x^{\prime}, y^{\prime}\right)=\frac{R_{c c}\left(x^{\prime}, y^{\prime}\right)}{\sum_{x, y} T(x, y)^{2} \cdot \sum_{x, y} I\left(x+x^{\prime}, y+y^{\prime}\right)^{2}}
$$


sendo,

$$
\begin{gathered}
R_{c c}\left(x^{\prime}, y^{\prime}\right)=\sum_{x, y}\left[T(x, y) \cdot I\left(x+x^{\prime}, y+y^{\prime}\right)\right]^{2} \\
T(x, y)=T(x, y)-\frac{1}{(w \cdot h)} \cdot \sum_{x^{\prime \prime}, y^{\prime \prime}} T\left(x^{\prime \prime}, y^{\prime \prime}\right) \\
I\left(x+x^{\prime}, y+y^{\prime}\right)=I\left(x+x^{\prime}, y+y^{\prime}\right)-\frac{1}{(w \cdot h)} \cdot \sum_{x^{\prime \prime}, y^{\prime \prime}} I\left(x^{\prime}+x^{\prime \prime}, y^{\prime}+y^{\prime \prime}\right)
\end{gathered}
$$

A escolha de uma imagem padrão adequada revela-se portanto um passo crucial para o sucesso deste método. Para imagem padrão deve ser escolhida uma imagem com um bom contraste entre as estruturas a identificar e com uma dimensão um pouco superior a um artefacto para ser possível identificar a sua forma circular em torno de um fundo uniforme. Neste estudo foi testada a utilização de uma imagem padrão criada artificialmente e uma imagem padrão extraída de uma das imagens do estudo. Verificou-se que usando a imagem real o método apresentava uma melhor taxa de detecção, sendo por isso a opção escolhida. A imagem padrão escolhida foi um reflexo bem contrastado e de contornos bem definidos de uma das imagens do estudo (figura 3.a). A imagem retirada é quadrada e inclui uma margem de pixéis de fundo em torno do reflexo.

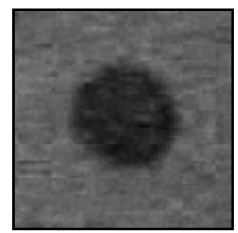

(a)

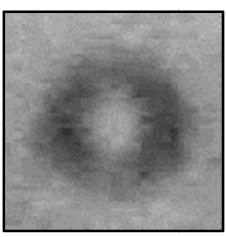

(b)

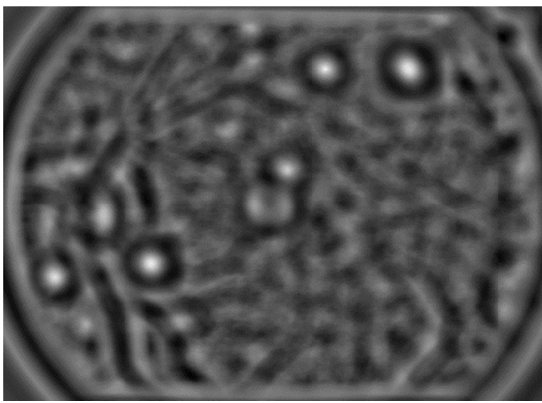

(c)

Figura 3 - Imagens padrão de reflexos luminosos (a) e do artefacto central (b);

c) Resultado da técnica de comparação de padrões entre a imagem 2.b e o padrão a).

O método proposto usa apenas uma imagem padrão uma vez que neste estudo as imagens foram todas adquiridas com o mesmo equipamento e apresentavam reflexos sempre com a mesma dimensão. No entanto, noutros estudos poderão ser incluídas outras imagens padrão adquiridas pelo equipamento em uso e ajustados os valores de limiar para detectar correctamente os novos artefactos.

Para identificar na imagem as regiões candidatas a ser classificadas como reflexos, o resultado da comparação de padrões é normalizado entre o e 255 sendo então aplicada uma binarização que assinala as regiões com intensidade superior a um limiar prédefinido (figura 4.a). O valor do limiar foi escolhido empiricamente variando o seu valor entre o e 255 e, uma vez que a taxa de verdadeiros negativos é desconhecida, foi analisada a curva Free-response ROC (FROC) (Chakraborty, 2000). Pelo gráfico 
apresentado na figura 4.b, o valor de limiar que se encontra mais próximo do canto superior esquerdo (sensibilidade máxima e menor taxa de falsas deteções) é o valor $t=194$, atingindo uma sensibilidade de $0.83 \mathrm{com}$ um rácio de 0.25 falsas deteções por imagem (FP/imagem)

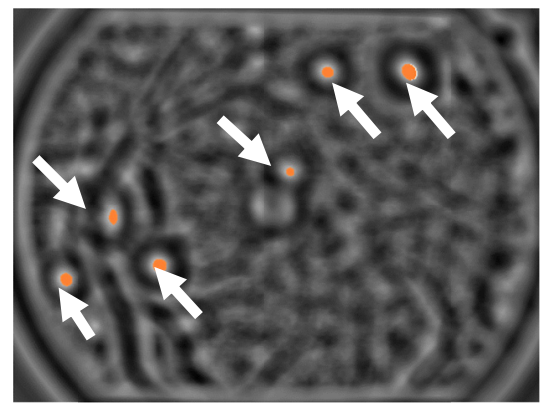

(a)

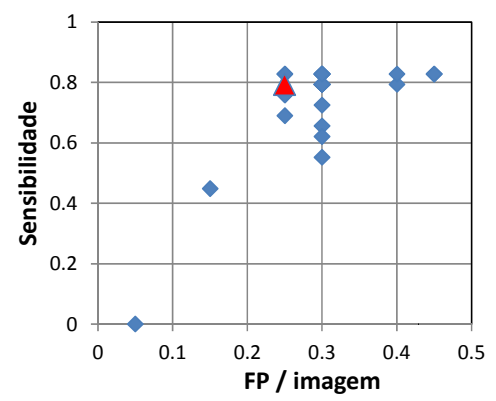

(b)

Figura 4 - a) Marcação dos candidatos com intensidade acima do limiar (a laranja);

b) Curva FROC para seleção do limiar de deteção de reflexos luminosos.

É de notar que é muito importante obter um correto compromisso entre sensibilidade e falsos positivos uma vez que os falsos positivos podem mascarar uma patologia e os falsos negativos podem confundir os sistemas de apoio ao diagnóstico que usem a imagem processada.

(1)

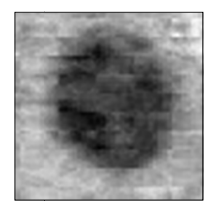

(2)

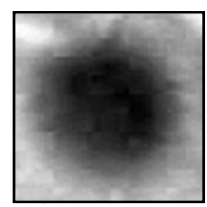

(3)

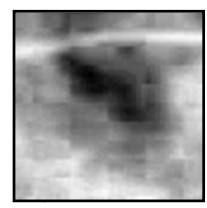

(4)

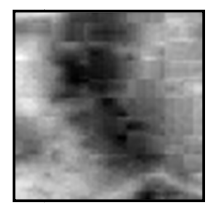

(a)
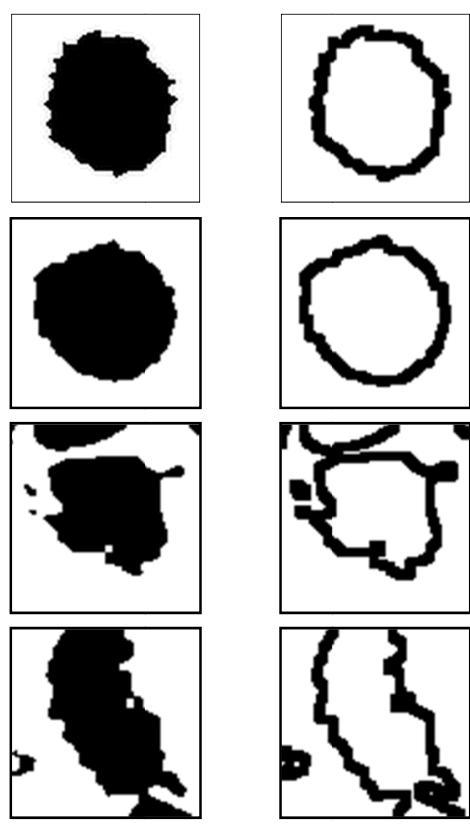

(b)
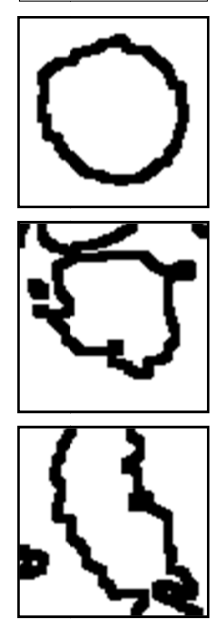

(c)

Figura 5 - Análise das imagens candidatas: (1 e 2) - candidatos verdadeiros; (3 e 4) candidatos falsos. a) canal de saturação; b) imagem binarizada; c) imagem de contornos. 
Embora o algoritmo de comparação de padrões tenha produzido uma boa segmentação dos candidatos a reflexo, gera um número significativo de falsas deteções requerendo uma pós-validação dos resultados. Uma vez que o disco ótico, devido à sua luminosidade, é uma das regiões mais propícias a ser confundida com um reflexo, qualquer candidato que se encontre nessa região será descartado.

Para efetuar a pós-validação dos candidatos foi treinado um classificador baseado em árvores de decisão, que usou como entradas algumas características dos candidatos, nomeadamente a sua forma, cor e semelhança com o padrão, as quais serão descritas em seguida.

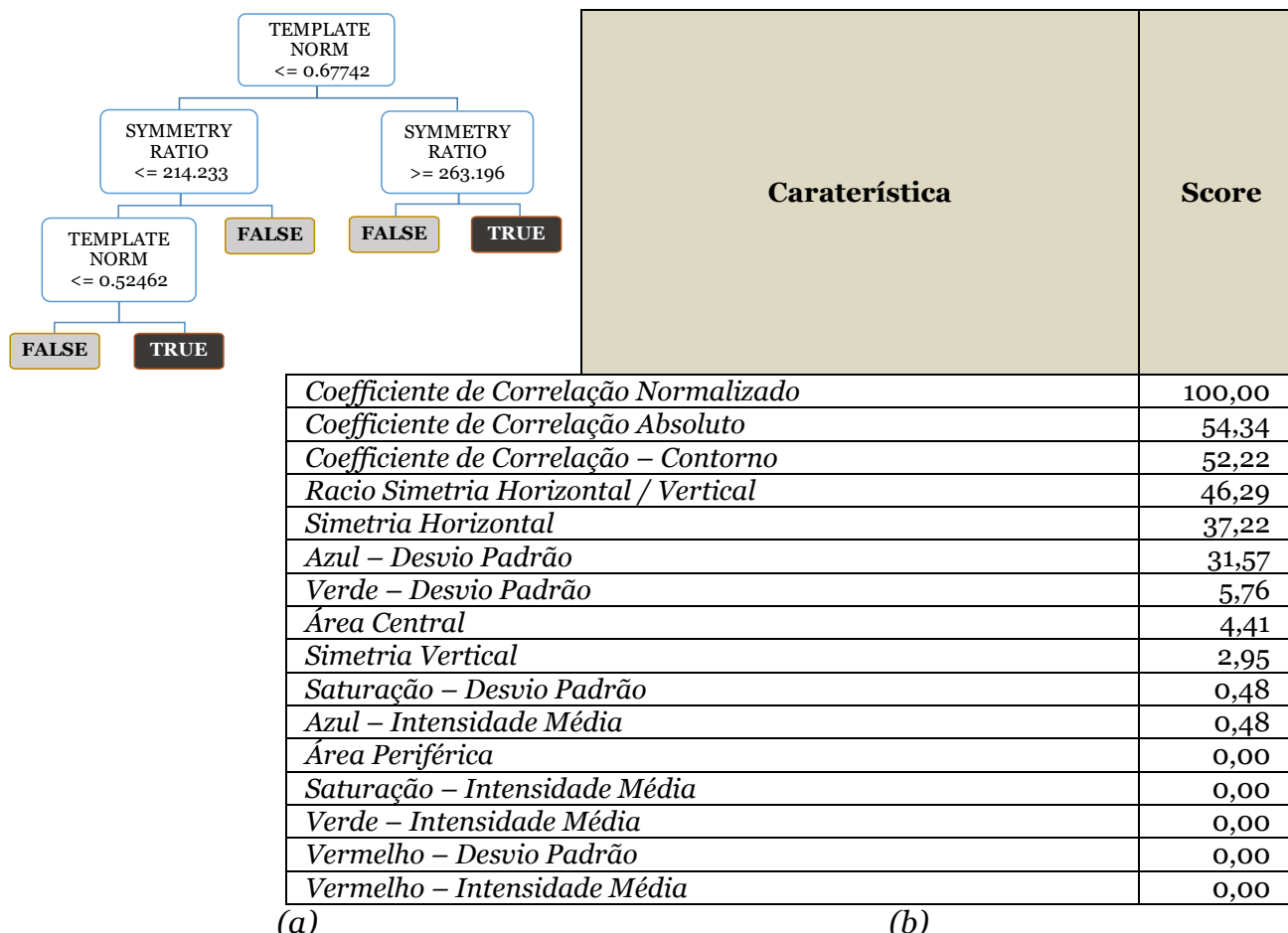

Figura 6 - (a) Importância das características atribuídas pelo software de geração de árvores de decisão; (b) Árvore de decisão gerada para validação dos candidatos.

Num primeiro passo os candidatos a reflexo são copiados da imagem de saturação para uma imagem com as mesmas dimensões da imagem padrão (figura 5.a) sendo registada a média e o desvio padrão do canal da saturação e dos três canais RGB, assim como os coeficientes de correlação normal (absoluto) e normalizado. Em seguida, é aplicada à imagem de saturação uma binarização pelo método de Otsu (Otsu, 1975) seguida de uma operação de fecho e outra de abertura (figura 5.b). Da imagem resultante é extraída a simetria horizontal e vertical e o número de pixéis a preto na zona central e periférica. Finalmente, é aplicado um filtro de Sobel para deteção de contornos (figura 5.c) sobre a qual é aplicada novamente a técnica de comparação de padrões com uma imagem padrão de um contorno circular. Na figura 5 é possível 
verificar que os candidatos verdadeiros $(1,2)$ apresentam uma forma circular regular e os candidatos falsos $(3,4)$ formas irregulares.

As caraterísticas extraídas da imagem foram aplicadas no treino e teste do classificador automático (figura 6.a), usando respetivamente 30\% e 70\% dos casos em cada uma das fases. Na figura 6.b são apresentadas todas as características usadas no treino do classificador e a sua importância de acordo com o software de geração de árvores de decisão.

Tal como esperado, os valores do coeficiente de correlação foram considerados os mais importantes sendo usados como testes iniciais da árvore de decisão. A simetria foi também considerada como muito importante, obtendo uma classificação superior às características relacionadas com a cor. Outras características de menor importância foram a área central e o desvio padrão dos canais azul e verde, sendo estes últimos usados para identificar falsos positivos em imagens a cores com fundos irregulares.

\subsection{Deteção do Artefacto Central}

Tal como os reflexos luminosos, o artefacto central pode ter uma influência negativa no desempenho de sistemas de diagnóstico automático. Como tal, é importante a sua identificação de forma a que essa região seja assinalada como danificada e/ou eventualmente alertar o operador para a necessidade de proceder à manutenção do equipamento. $\mathrm{O}$ artefacto central surge sempre no centro da imagem apresentando uma cor esbranquiçada e uma forma toroidal distinta de qualquer outra estrutura retiniana.

A metodologia adotada para a deteção deste artefacto é muito similar à anterior, sendo também utilizada a técnica de comparação de padrões com o coeficiente de correlação normalizado. Uma vez que este artefacto surge sempre na zona central da imagem, a pesquisa foi restringida apenas a essa zona. A imagem padrão utilizada no estudo foi igualmente retirada das imagens disponíveis, não contendo patologias e apresentando um bom contraste com o fundo (figura 3.b).

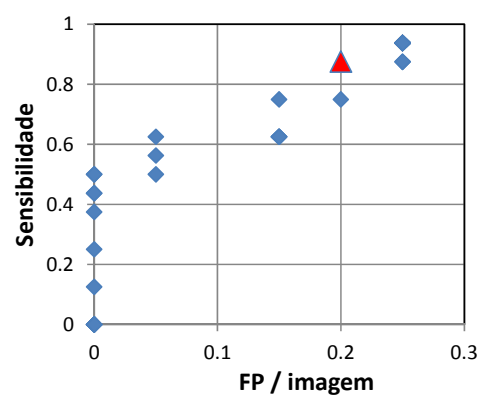

Figura 7 - Curva FROC para seleção do limiar de deteção do artefacto central.

Para a deteção e validação do artefacto central é analisado apenas o valor do coeficiente de correlação normalizado tendo sido definido empiricamente um limiar de $t=0.475$ 
acima do qual este é aceite. Este limiar foi também escolhido através da curva FROC (figura 7) usando os mesmos critérios utilizados para os reflexos luminosos. Obteve-se assim uma sensibilidade de 0.88 e um rácio de 0.2 falsas deteções por imagem. Uma vez que a forma do artefacto central é bem definida e que a região de pesquisa é de pequenas dimensões, o número de classificações incorretas revelou-se bastante baixo.

\section{Resultados}

O método proposto para a deteção dos reflexos luminosos e do artefacto central foi implementado em linguagem $\mathrm{C} \#$ usando a biblioteca de funções OpenCV Image Processing Library. Tal como previamente explicado, o disco ótico foi assinalado manualmente nas imagens sendo descartado qualquer artefacto detetado dentro desta área.

Na figura 8 podem ser observados dois exemplos de deteção de artefactos. Na figura 8.a podemos ver uma imagem de qualidade normal na qual estão indicados os artefactos detetados. A figura 8.b mostra que, mesmo numa imagem com qualidade deficiente, é possível detetar corretamente todos os artefactos nela presentes. Os dois algoritmos de deteção de artefactos foram avaliados usando os conceitos de sensibilidade e de falsas deteções em cada uma das imagens do conjunto de treino e do conjunto de teste (ver tabela 1). Apesar do bom resultado obtido na detecção de reflexos luminosos com uma sensibilidade de 0.83 e um valor de falsos positivos de apenas 0.25 por imagem, foram encontradas algumas limitações relacionadas com a localização dos reflexos luminosos. Foi constatado que o algoritmo de comparação de padrões apresenta algumas dificuldades na detecção de artefactos localizados na periferia das imagens o que se traduziu numa redução da sua eficiência especialmente sobre as imagens do conjunto de teste.

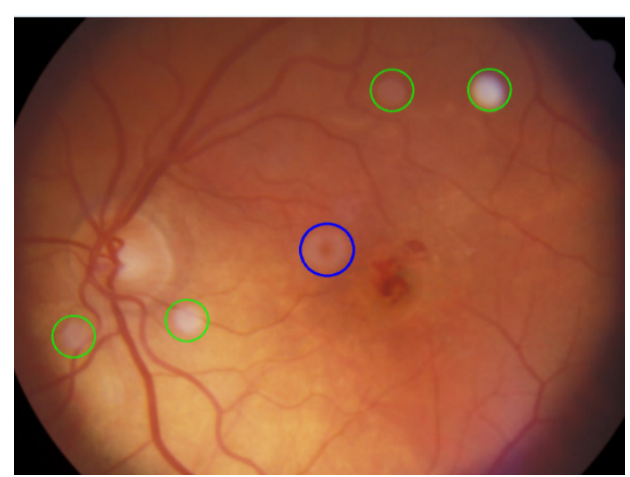

(a)

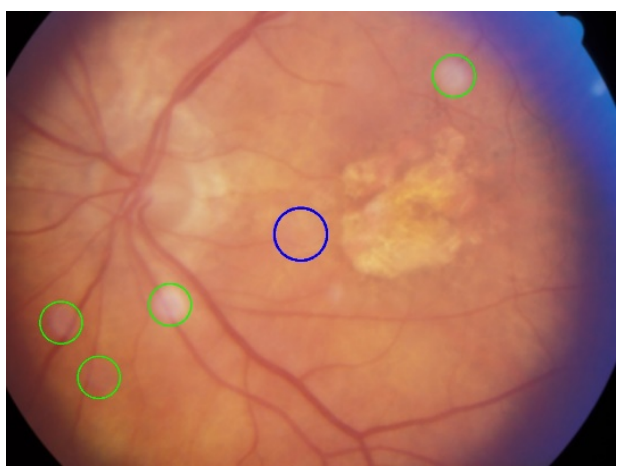

(b)

Figura 8 - Exemplos de reflexos luminosos (a verde) e de artefactos centrais (a azul escuro) marcados sobre imagens da retina de qualidade normal $(a)$ e baixa $(b)$. 
As imagens com opacidade significativa, causada por cataratas, dificultam o processamento reduzindo a taxa de sucesso dos algoritmos. É, no entanto, de salientar que em nenhum caso se verificou qualquer confusão entre artefactos e lesões ou estruturas da retina.

Tabela 1 - Resultados para o conjunto de treino e de teste.

\begin{tabular}{|l|c|c|c|c|c|}
\cline { 2 - 5 } \multicolumn{1}{c|}{} & \multicolumn{2}{c|}{ Treino } & \multicolumn{2}{c|}{ Teste } \\
\cline { 2 - 5 } \multicolumn{1}{c|}{} & Sensibilidade & FP/Imagem & & Sensibilidade & FP/Imagem \\
\hline Artefacto Central & 0.88 & 0.20 & & 0.97 & 0.12 \\
\hline Reflexos Luminosos & 0.83 & 0.25 & & 0.73 & 0.36 \\
\hline
\end{tabular}

A deteção do artefacto central obteve uma sensibilidade de 0.97 com uma taxa de falsos positivos/imagem de 0.12. Os melhores resultados na deteção do artefacto central devem-se ao facto deste ter uma posição pré-determinada e de apresentar um formato muito característico. A comparação de resultados com outros trabalhos não foi possível por não terem sido encontrados até à data trabalhos similares que permitam a sua comparação. A utilização de bancos de imagens da retina de referência tais como o INSPIRE (Meindert Niemeijer et al., 2011), o STARE (Hoover et al., 2000) ou o DRIVE (Staal et al., 2004) foi também impossível uma vez que as imagens que os compõem foram previamente selecionadas de forma a não conterem imagens com artefactos. Embora possam ser encontrados na literatura alguns trabalhos referindo o problema dos artefactos nas imagens da retina, nenhum dos estudos conhecidos até à data propõe a sua deteção automática.

Para aplicação desta metodologia a um cenário real prevê-se que apenas seja necessário escolher novas imagens padrão adquiridas pelo equipamento em uso e ajustar o valor do limiar de deteção de artefactos por forma a detectar artefactos que sejam visíveis e que possam ser confundidos com alguma patologia retineana.

\section{Conclusões}

Neste artigo é apresentada uma metodologia para a deteção de artefactos baseada nas suas características de cor e forma. O objetivo principal deste estudo é excluir de estudos posteriores ou alertar automaticamente para a necessidade de repetição da captação sempre que as imagens apresentem problemas significativos. É assim possível por um lado aumentar a eficiência das ações de rastreio ao baixar o número de pacientes que têm de voltar a sujeitar-se a exames e por outro lado melhorar a performance dos sistemas de análise automática de patologias ao evitar que imagens ou zonas das imagens contendo artefactos sejam processadas.

A metodologia proposta utiliza técnicas de comparação de padrões para pesquisar potenciais candidatos a artefactos na imagem da retina. Dado que esta técnica tem tendência para detetar um número excessivo de candidatos foi também implementado um processo de validação baseado em árvores de decisão que permite excluir falsos candidatos. A metodologia proposta apresenta uma baixa complexidade computacional permitindo a sua integração num sistema de deteção de patologias sem um impacto significativo. 
A sensibilidade e a taxa de falsos positivos obtidos pelo sistema (0.97/0.12 para o artefacto central e 0.73/0.36 para a deteção do artefacto central) mostram que a metodologia proposta permite obter resultados muito aceitáveis mesmo na presença de imagens de reduzida qualidade provenientes de pacientes afetados por cataratas.

Os próximos passos deste trabalho passarão pelo teste da metodologia proposta com um conjunto de imagens mais alargado e pela sua introdução num sistema de rastreio oftalmológico com deteção de artefactos em tempo real.

Tendo como base a revisão de literatura efetuada podemos afirmar que a metodologia proposta neste trabalho é inovadora e prometedora para a deteção de artefactos em imagens da retina. A introdução deste tipo de algoritmos poderá, no futuro próximo, aumentar a eficiência dos sistemas de rastreio e diagnóstico de doenças oculares o que virá certamente contribuir para a melhoria das condições de vida da população em geral e em especial da sua faixa sénior.

\section{Referências}

Azemin, M. Z. C., Kumar, D. K., Wong, T. Y., Kawasaki, R., Mitchell, P., \& Wang, J. J. (2011). Robust methodology for fractal analysis of the retinal vasculature. IEEE Transactions on Medical Imaging, 30(2), 243-50.

Bertalmio, M., Sapiro, G., Caselles, V., \& Ballester, C. (2000). Image inpainting. In Proceedings of the 27th annual conference on Computer graphics and interactive techniques - SIGGRAPH 'oo (pp. 417-424). New York, New York, USA: ACM Press.

Brunner, S., Mora, A., Fonseca, J., Weber, T., Falkner-Radler, C. I., Oeser, R., \& Binder, S. (2013). Monitoring of drusen and geographic atrophy area size after cataract surgery using the MD3RI tool for computer-aided contour drawing. Ophthalmologica, 229(2), 86-93.

Burke, M. W. (1996). Image acquisition (p. 917). Chapman \& Hall.

Chakraborty, D. P. (2000). The FROC, AFROC and DROC Variants of the ROC Analysis. In R. L. Van Metter, J. Beutel, \& H. L. Kundel (Eds.), Handbook of Medical Imaging, Volume 1. Physics and Psychophysics (pp. 771-796). 1000 20th Street, Bellingham, WA 98227-0010 USA: SPIE.

Davis, H., Russell, S., Barriga, E., Abramoff, M., \& Soliz, P. (2009). Vision-based, realtime retinal image quality assessment. In 2009 22nd IEEE International Symposium on Computer-Based Medical Systems (pp. 1-6). IEEE.

Gu, J., Ramamoorthi, R., Belhumeur, P., \& Nayar, S. (2009). Removing image artifacts due to dirty camera lenses and thin occluders. ACM Transactions on Graphics, 28(5), 1 .

Hamilton, A. M. P., Gregson, R., \& Fish, G. E. (1998). Text atlas of the retina (p. 417). Butterworth-Heinemann. 
Hoover, A., Kouznetsova, V., \& Goldbaum, M. (2000). Locating blood vessels in retinal images by piecewise threshold probing of a matched filter response. IEEE Trans Med Imaging, 19(3), 203-210.

Mora, A. D., Vieira, P. M., Manivannan, A., \& Fonseca, J. M. (2011). Automated drusen detection in retinal images using analytical modelling algorithms. Biomedical Engineering Online, 10, 59.

Niemeijer, M., van Ginneken, B., Russell, S. R., Suttorp-Schulten, M. S., \& Abramoff, M. D. (2007). Automated detection and differentiation of drusen, exudates, and cotton-wool spots in digital color fundus photographs for diabetic retinopathy diagnosis. Invest Ophthalmol Vis Sci, 48(5), 2260-2267.

Niemeijer, M., Xu, X., Dumitrescu, A. V, Gupta, P., van Ginneken, B., Folk, J. C., \& Abramoff, M. D. (2011). Automated measurement of the arteriolar-to-venular width ratio in digital color fundus photographs. IEEE Transactions on Medical Imaging, 30(11), 1941-50.

Otsu, N. (1975). A threshold selection method from gray-level histograms. Automatica, 11, 285-296.

Pinão, J., Oliveira, C. M., Mora, A., \& Dias, J. (2013). Detection of Anatomic Structures in Retinal Images. In J. M. R. S. Tavares \& R. M. N. Jorge (Eds.), Topics in Medical Image Processing and Computational Vision (p. 300). Springer Science+Business Media Dordrecht.

Porte, C. (2012). Pathogenesis and Management of Age-Related Macular Degeneration. Scottish Universities Medical Journal, 1(2), 141-153.

Rogers, A. H. (2007). Retina (p. 288). Elsevier Health Sciences.

Staal, J., Abràmoff, M. D., Niemeijer, M., Viergever, M. A., \& van Ginneken, B. (2004). Ridge-based vessel segmentation in color images of the retina. IEEE Transactions on Medical Imaging, 23(4), 501-509.

Sun, J., Yuan, L., Jia, J., \& Shum, H.-Y. (2005). Image completion with structure propagation. ACM Transactions on Graphics, 24(3), 861.

United Nations. (2013). World Population Ageing 2013 (No. ST/ESA/SER.A/348).

Wilson, M. P., Yang, S., Mitra, S., Raman, B., Nemeth, S. C., \& Soliz, P. (2003). Full automation of morphological segmentation of retinal images: a comparison with human-based analysis. In M. Sonka \& J. M. Fitzpatrick (Eds.), SPIE 5032, Medical Imaging 2003: Image Processing (pp. 27-37).

Yu, H., Agurto, C., Barriga, S., Nemeth, S. C., Soliz, P., \& Zamora, G. (2012). Automated image quality evaluation of retinal fundus photographs in diabetic retinopathy screening. In 2012 IEEE Southwest Symposium on Image Analysis and Interpretation (pp. 125-128). IEEE.

Zhou, C., \& Lin, S. (2007). Removal of Image Artifacts Due to Sensor Dust. In 2007 IEEE Conference on Computer Vision and Pattern Recognition (pp. 1-8). IEEE. 